\title{
2 Konzeptueller Rahmen zur Simultanität multimodaler Aktivitäten
}

Um die interaktionalen Verfahren und kommunikativen Funktionen der Bearbeitung gleichzeitiger Relevanz von multiplen Aktivitäten durch die Beteiligten analysieren zu können, sollen zunächst der Forschungsstand und die aktuellen Desiderate der multiactivity-Forschung dargestellt werden (2.1). Wie dabei deutlich werden wird, bauen die dort genannten Studien auf einem breiten Feld interaktionsanalytischer Arbeiten auf, die das Verständnis von Zeitlichkeit für die Organisation sozialer Situationen in Interaktionen maßgeblich bestimmen: das sequentiell geordnete Nacheinander der Interaktionsbeiträge einerseits und die Simultanität multimodaler Ressourcen andererseits (2.2). Der analytische Zugriff auf multiple Aktivitäten in Interaktionen im Rahmen dieser Studie macht es darüber hinaus notwendig, die teilweise widersprüchlichen Konzeptualisierungen des Aktivitätsbegriffs in der ethnomethodologischen Konversationsanalyse $\mathrm{zu}$ systematisieren und daraus das für diese Arbeit geltende Verständnis von Aktivität abzuleiten (2.3). Damit liefert dieses Kapitel einen Überblick über den aktuellen Forschungsstand zum Untersuchungsgegenstand und spannt den für diese Studie geltenden konzeptuellen Rahmen auf.

\subsection{Stand der Forschung zum Phänomen multiactivity}

Aufgrund der vielen Aktivitäten, die während einer Interaktion zeitgleich ablaufen können, stehen Interagierende oftmals vor der Herausforderung, ihre Beteiligungsweisen an laufende Aktivitäten anzupassen. Dieses im Folgenden als multiple Aktivitäten bezeichnete interaktionale Phänomen kann in vielen institutionellen und arbeitsgebundenen Settings beobachtet werden. Goodwin \& Goodwin (1996) arbeiten beispielsweise heraus, wie mehrere Arbeitsaktivitäten in einem Flughafenkontrollraum interaktiv miteinander verbunden werden. Für Arzt-Patientengespräche beschreiben Robinson \& Stivers (2001) die Praktiken, mit denen die Beteiligten ANAMNESE-Aktivitäten mit den ScHREIB-Aktivitäten der Ärzte koordinieren. Pitsch (2007) untersucht, wie UNTERRICHTSDISKURS und MITSCHREIBEN als aufeinander bezogene, parallele Aktivitäten organisiert werden. Bei Mortensen (2008) stehen ebenfalls UNTERRICHTSDISKURSE im Forschungsinteresse, allerdings vorrangig die Involvierung der Schüler^innen in GESPRÄCHEN mit ihren Mitschüler^innen während des UnTERRICHTS. Anhand von Videodaten in einem Call Center arbeitet Mondada (2008) heraus, dass multiple Aktivitäten sich entweder autonom verhalten oder zusammenhängen können, wobei 
sich der jeweilige Autonomie-Status im Verlauf der Interaktion durchaus ändern kann. Im Setting der Notfallübungen zeigt Deppermann (2014b), wie gleichzeitig verbal INFORMIERT wird, während simultan Gestik zur Teilnahme an einer gemeinsamen Aktivität (ANWEISEN, EINEN PATIENTEN ZU BEHANDELN) eingesetzt wird. Arminen, Koskela \& Palukka (2014) betrachten die multiplen Aktivitäten eines Flugverkehrkontrolldienstes, die miteinander koordiniert werden müssen, um Flugzeuge sicher starten und landen zu lassen. Weidner (2017) schließlich untersucht, wie KocH-Aktivitäten in Kochshows mit anderen Aktivitäten (INFORMIEREN, BERICHTEN, ERZÄHLEN, INSTRUIEREN etc.) verbunden werden. Multiple Aktivitäten sind darüber hinaus nicht auf institutionelle Kontexte beschränkt. Egbert $(1993,1997)$ zeigt anhand von Videodaten in privaten Umgebungen, wie sich laufende Unterhaltungen (u.a. BEWERTUNGEN und ERzÄHLUNGEN) auf mehre Interaktionen aufteilen (schisming), während die Interagierenden zugleich ESSEN oder KAFFEE TRINKEN. Für Interaktionen während des Einrichtens einer Wohnung nach einem Umzug beschreiben Krafft \& Dausendschön-Gay (2007) verschiedene Koordinierungsprozesse mehrerer alltäglicher Aktivitäten (z. B. DAS WEITERE VORGEHEN VORSCHLAgEN, während des WEGRÄUMENS EINES STAUBSAUGERS oder KAFFEE TRINKEn beim Schrank AufBauen). Für die Auswahl dieser Studien gilt, dass sie die interaktionalen Phänomene der multiplen Aktivitäten und die für die Beteiligten daraus erwachsenen Herausforderungen explizit zum Forschungsgegenstand machen. Obwohl sie zeigen, dass multiple Aktivitäten weder in institutionellen noch in alltäglichen Settings einen Sonderfall darstellen, bilden sie dennoch als Forschungsgegenstand innerhalb der konversationsanalytischen Forschungslandschaft eine Ausnahme. Grund dafür ist der Fokus der Konversationsanalyse auf Praktiken der Sequenzorganisation einzelner Aktivitäten („monoactivity“, Haddington et al. 2014: 21), sodass ein Blick über die Sequenzgrenzen der betrachteten Monoaktivitäten nur selten notwendig wird. Die Analysen dieser Arbeit zeigen, dass Interagierende bei der Koordination multipler Aktivitäten auf die gleichen Verfahren zurückgreifen, die sie auch zur Herstellung multimodaler Interaktionsbeiträge verwenden. Der Unterschied zwischen dem Vollzug einzelner oder multipler Aktivitäten zeigt sich vielmehr in den Konsequenzen, die diese Verfahren für die jeweiligen Interaktionen haben können. Wenn beispielsweise mithilfe einer Delay-Organisation (Deppermann \& Schmitt 2007) im Rahmen einer Monoaktivität die Ausführung einer einzelnen Ressource (z. B. eine Geste) verzögert wird, kann ein Delay bei einer Multiaktivität ein ganzes Ressourcenbündel verzögern. Das kann unter anderem Auswirkungen auf die Realisierung einer damit verbundenen Aktivität haben, die daraufhin abgebrochen oder pausiert werden kann, was sich wiederum in der Teilnehmendenrahmung oder eine Priorisierung einer der gleichzeitig relevanten Aktivitäten niederschlägt. 
Da in interaktionsanalytischen Arbeiten der letzten Zeit immer öfter mehrere simultane Handlungsverläufe in den Analysefokus genommen werden, ergeben sich neben einem tieferen Verständnis sozialer Situationen und neuen Perspektiven auf Priorisierung und Sequentialität von multiplen Aktivitäten auch neue methodologische Hürden. Die zentrale Herausforderung bei der Analyse multipler Aktivitäten ist für Forschende die Frage: Für welche Teilnehmenden sind die beobachteten Aktivitäten multiple Aktivitäten? Videodaten erlauben es Interaktionsforschenden mitunter mehr Aktivitäten in einer Situation zu beobachten, als die Teilnehmenden der Situation selbst wahrnehmen können. Eine solches Auftreten von zwei oder mehr Aktivitäten in einer Situation wird in einigen interaktionsanalytischen Studien als parallele Aktivitäten konzeptualisiert (Pitsch 2006, 2007; Koole 2007; Mondada 2008; Mortensen 2008, 2013; Arminen, Koskela \& Palukka 2014). Das namensgebende Prinzip der Parallelität ergibt sich aus der etischen Beobachtung der Situation. Gleichzeitigkeit als Parallelität umfasst dabei alle Aktivitäten einer Situation und deren temporale bzw. koordinative Relation zueinander. Bei einer Betrachtung der Parallelität stellt sich die Frage, wie parallele Aktivitäten in einer konstanten Koordinierungsleistung der Interagierenden simultan miteinander in Bezug gesetzt und sequentiell aufeinander abgestimmt werden. Das heißt, ein ${ }^{\star}$ e Interagierende ${ }^{\star}$ r koordiniert parallele Aktivitäten, wenn er^sie eine Aktivität vollzieht ohne sich an einer weiteren (parallelen) Aktivität der Situation zu beteiligen (parallel but separate activities, Hochuli 2019: 470). In den oben genannten Studien treten innerhalb eines Settings zur gleichen Zeit unterschiedliche Aktivitäten auf. Einige davon stehen in koordinativer Wechselwirkung miteinander, für andere Aktivitäten ist ein solches Verhältnis, das über die bloße Konstitution einer Situation hinausgeht, nicht festzustellen. Daraus ergibt sich, dass das Vorhandensein verschiedener Aktivitäten in der Wirkzone einer`s Teilnehmenden (Schütz \& Luckmann 2017 [1979]) nicht ausreicht, um für eine Person die gleichzeitige Relevanz multipler Aktivitäten anzunehmen. Stattdessen wird die intersubjektive Wahrnehmung von Gleichzeitigkeit erst dann erkennbar, wenn Interagierende ihr koordinatives Verhalten darauf ausrichten. Der Begriff parallele Aktivitäten scheint daher nicht treffend, wenn man die gleichzeitige Involvierung der Teilnehmenden in eine für alle Beteiligten relevanten Aktivität betrachten will. Eine solche Ausrichtung der Teilnehmenden einer Situation auf dieselbe Aktivität ist leicht bestimmbar, wenn sich Interagierende verbal an einer Aktivität beteiligen. Handelt es sich hingegen um sprachfreie Beteiligungsweisen, ist die gleichzeitige Relevanz analytisch schwerer zu fassen (vgl. dazu Kap. 3). Aufgrund der langjährigen Fokussierung der Interaktionsforschung auf verbale Phänomene werden häufig Diskursaktivitäten mit verbalem Anteil automatisch als Hauptaktivität betrachtet, während manuelle Aktivitäten mit „verbaler Abstinenz“ (Heidtmann \& Föh 2007) analytisch lediglich als Neben- 
aktivitäten behandelt werden (vgl. Goodwin 1986). Diese hierarchische Vorstellung von Aktivitäten findet sich schon bei Goffman (1963: 20-21), der Aktivitäten in „main activities“ und ,side activities“ einteilt. Grund für diese Unterscheidung ist bei ihm der soziale Anlass. Handeln Teilnehmende auf diesen Anlass bezogen, zeigen sie übergeordnete Involvierung (,dominant involvement“) an und realisieren eine Hauptaktivität. Ist die Aufmerksamkeit auf den sozialen Anlass kurzzeitig nicht notwendig, kann eine Nebenaktivität mit untergeordneter Involvierung („subordinate involvement“) realisiert werden. Diese Verteilung verändert sich, wenn eine auf den sozialen Anlass bezogene Aktivität ,automatically and unthinkingly“ realisiert werden kann:

Typically, it is expected that a main involvement will be a dominating one and a side involvement a subordinate one, as when a worker smokes a cigarette unthinkingly but only when and where the job allows. This relationship, however, is by no means invariable. Many dominating involvements, such as work tasks, can be sustained automatically and unthinkingly for long periods, allowing the individual to devote his main focus of attention to pursuits such as idle gossip, which, however involving, will be put aside when the task requires attention.

(Goffman 1963: 43-45)

Innerhalb der konversationsanalytischen Methodologie ist es unmöglich, Aussagen über den mentalen Status von Interagierenden zu treffen. Ob eine Aktivität also „gedankenlos und automatisch“ oder „bewusst“ hergestellt wird, kann aus konversationsanalytischer Perspektive kein Kriterium sein. Für Forschende und Interagierende gleichermaßen zugänglich sind lediglich die multimodalen Ressourcen, mit denen Teilnehmende anzeigen, zu welchem Grad sie sich auf eine bestimmte Aktivität hin orientieren. Damit unterscheiden sich nicht die Aktivitäten selber in Bezug auf die hierarchische Struktur, sondern die Beteiligungsweisen der Teilnehmenden, mit denen sie einander ihre interaktionale Zugehörigkeit zu einer bestimmten Aktivität anzeigen. Dies bedeutet für die Betrachtung multipler Aktivitäten, dass nicht gefragt wird, welche Aktivität „wichtiger“ oder „unwichtiger“ für den Interaktionsverlauf ist, sondern auf welche Involvierungspraktiken Teilnehmende zurückgreifen, um eine gleichzeitige Beteiligung an zwei (oder mehr!) Aktivitäten zu ermöglichen. Damit spielt das populäre Konzept Multitasking ${ }^{5}$ in dieser Arbeit keine Rolle. Bei diesem wird vor allem in Laborsettings (z. B. Yang, Heeman \& Kun 2011) untersucht, auf welche Weise Menschen unterschiedliche Aufgaben bearbeiten und welche kognitiven Prozesse dabei ablaufen. Die Konzeption von Gleichzeitigkeit leitet sich dabei von den Aufgaben ab, die von den Studienleitungen so konzipiert wurden, dass die Teilnehmenden diese

5 Für eine ausführlichere Diskussion von „Multitasking“ aus Perspektive multipler Aktivitäten siehe Haddington et al. (2014: 5). 
zur gleichen Zeit bearbeiten. Das dieser Studie zugrundeliegende Datenmaterial einer teilnehmenden Beobachtung an Theaterproben würde es ermöglichen, anhand des ethnographischen Wissens für die einzelnen Beteiligten detaillierte Arbeits- und Aufgabenbeschreibungen anzufertigen. Das kann leicht dazu verführen, jegliches Handeln der Theaterschaffenden mit ihren Jobbeschreibungen in Verbindung zu bringen und als aufgabenspezifische Handlungen $\mathrm{zu}$ interpretieren. Doch auch wenn den Forschenden die Aufgabenbereiche der Teilnehmenden bekannt sind, kann daraus nicht ohne Weiteres geschlossen werden, welche Aufgabe den Theaterschaffenden zu einem spezifischen Zeitpunkt tatsächlich präsent ist. So ist es beispielsweise eine bekannte Aufgabe („task“) der Regieassistentin, Textänderungen mitzuschreiben. In vielen Situationen ist daher beobachtbar, dass die Regieassistentin auf ihren Block schaut und den Stift bewegt. Doch erst beim Sichten der Eye-Tracking-Daten oder der Arbeitsdokumente wird deutlich, dass die Regieassistentin in einigen Fällen Blumen und Häuser auf den Blockrand gekritzelt hat. Das Wissen um eine teilnehmendenspezifische Aufgabe ist demnach analytisch nicht gleichzusetzen mit der tatsächlich durch die Teilnehmenden realisierten Aktivität. Deshalb ist es für Analysen sinnvoller, sich auf die beobachtbaren Realisierungsformen zu konzentrieren und die institutionellen Aufgabenbeschreibungen erst dann in der Analyse heranzuziehen, wenn die Teilnehmenden dieses Wissen relevant setzen. Daher gilt: Tasks als zielorientierte Einheiten werden durch Aktivitäten erfüllt. Beim Multitasking gibt es also mehrere Aufgaben, die mehrere Aktivitäten erfordern können. Möglich ist auch der umgekehrte Fall: Zwei Tasks werden durch dieselbe Aktivität realisiert. Multitasking kann somit die Koordinierung multipler Aktivitäten erfordern; multiple Aktivitäten müssen aber nicht zwangsläufig Multitasking-Phänomene sein.

Von Tasks abzugrenzen sind interaktionskonstituierende Aufgaben, sogenannte Interaktionsaufgaben. Sie umfassen interaktionale Daueranforderungen „wie Herstellung von Kopräsenz, wechselseitige Identifizierung und wechselseitigem face-work im bestätigenden Austausch“ (Auer 2020: 77). In der Interaktionsforschung wird Interaktion daher verstanden als „komplexe Hierarchie von Aufgaben“ (Fiehler 1990: 29), mit denen Interagierende ihre soziale Beziehung und Identität bearbeiten, die soziale Situation herstellen und die Handlungszusammenhänge konstituieren. Indem sich Interagierende an einer Aktivität beteiligen, gehen sie „Aktivitätsverpflichtungen“ (Kallmeyer 1981: 96) ein, denen sie im Zuge der Bearbeitung der Interaktionsaufgaben nachkommen müssen. Werden Interaktionsaufgaben nicht oder unzureichend bearbeitet, steigt „das Risiko von Turbulenzen in der Interaktion bis hin zum Scheitern der gemeinsamen Handlungen“ (ebd.). Wie die Analysen in den Kapitel 5-7 zeigen werden, nehmen Interaktionsaufgaben bei der Betrachtung gleichzeitigen Relevanz multipler Aktivitäten eine besondere Stellung ein. Denn in solchen Situationen müssen Beteiligte mitunter 
mehrere Interaktionsaufgaben unterschiedlicher Aktivitäten bearbeiten. So kann in einigen Fällen der Abbruch einer Aktivität zugunsten einer ko-relevanten anderen Aktivität auch auf eine fehlende bzw. für die Interaktion unzureichende Bearbeitung anstehender Interaktionsaufgaben (z.B. fehlender wechselseitiger Blickkontakt) zurückgeführt werden.

Das Phänomen der gleichzeitigen Relevanz multipler Aktivitäten wird in der internationalen Forschungsgemeinschaft unter dem Begriff multiactivity (Haddington et al. 2014) behandelt. Unter dieser Bezeichnung werden bislang solche Interaktionsanalysen subsumiert, die eine (wie auch immer geartete) Simultanität mehrerer Aktivitäten in Interaktionen untersuchen (Nishizaka 2014; Mondada 2014c; Hoey 2015, 2018b). Das Phänomen multiactivity umfasst Praktiken bei der Koordination komplexer Aktivitätsverbünde, bei denen Teilnehmende die Involvierung und „Ko-Relevanz“ (Haddington et al. 2014: 3) zweier (oder mehrere) simultan realisierter Aktivitäten aufrechterhalten müssen. Dabei folgen multiactivities ihren eigenen temporalen Logiken, die so miteinander abgestimmt werden, dass eine simultane Koordinierung möglich wird (Mondada 2008). Mithilfe simultaner Koordinierungspraktiken verbinden Interagierende zwei oder mehr Handlungsverläufe (=Aktivitäten) zu einer interaktionalen Einheit (=Multiaktivität) (Mondada 2011: 207), bis dieselben Ressourcen für verschiedene Aktivitäten benötigt werden (Ticca 2014: 210). Multiaktivitäten erhöhen die Komplexität von Face-to-Face-Interaktionen, weil Teilnehmende unterschiedliche multimodale Ressourcen für verschiedene Handlungsverläufe mit eigenen sequentiellen Ordnungen verwenden (Mondada 2014c: 38) und stellen besondere Anforderungen für alle Teilnehmenden in Interaktionen dar. Diese Anforderungen werden in der vorliegenden Arbeit vor allem auf der koordinativen (und nicht auf einer kognitiven oder inhaltlichen) Ebene betrachtet als strukturelle Bedingungen, die zum Bearbeiten einer Interaktionsaufgabe erfüllt sein müssen. Dies ist eine der Voraussetzungen dafür, dass Interaktion als wechselseitig aufeinander ausgerichtete Kommunikationsbeiträge möglich wird, wie Schegloff in der Einleitung zu Sacks (1992: lvi) Lectures formuliert: „What that has required of recipients, and how those requirements are formative of their talk in turn“. Anstelle von talk stehen in dieser Studie nun die multimodalen Koordinierungsbedingungen im Fokus, auf sich Beteiligte beziehen, wenn ko-relevante Aktivitäten gleichzeitig vollzogen werden. Solche Multiaktivitäten treten dann auf, wenn Interagierende zwei oder mehr separate, wahrnehmbare und gleichermaßen relevante Projekte (Licoppe \& Tuncer 2014: 169) bearbeiten müssen, z. B. einen Tanz vorzuführen, während auf eine Besonderheit des Tanzes hingewiesen wird (siehe Kapitel 6). Es wird deutlich, dass Multiaktivitäten keine zufällig auftretenden, anforderungsreichen Phänomene sind, mit denen Interagierende umgehen müssen. Stattdessen sind sie einerseits eine „kollektive, kollaborative und intersubjektive“ Herstellungsleistung 
des gesamten Interaktionsensembles in situ (Haddington et al. 2014: 6) und andererseits eine interaktionale Lösung dafür, mehrere simultan relevante Interaktionsaufgaben gleichzeitig bearbeiten zu können. Die Analyse der gleichzeitigen Involvierung in verschiedene Teil-Aktivitäten einer Multiaktivität ermöglicht einen Blick auf die Organisation der Relevantsetzungen der Interagierenden innerhalb einer Situation, die sie mitgestalten und auf die sie sich konstant beziehen (vgl. Goodwin 2000d: 178). Da die Involvierung einer Person in eine Multiaktivität nicht von den multiplen Aktivitäten anderer Beteiligter separiert werden kann, sind neben den priorisierten Teilnehmerprojekten auch adaptive Praktiken der Teilnehmerrahmungen und differenzierte Beteiligungsweisen beobachtbar. Multiaktivitäten sind daher ein soziales Phänomen, das die inter- und intrapersonelle Koordination (Deppermann \& Schmitt 2007) aller Beteiligten erfordert und die Organisation sozialer Interaktion maßgeblich beeinflusst.

Das gleichzeitige Auftreten der Aktivitäten wird von der multiactivity-Forschung oftmals unter dem Gesichtspunkt des Auflösens der Simultanität durch die Interagierenden betrachtet. Dazu gehören Phänomene des Abbrechens (Keisanen, Rauniomaa \& Haddington 2014; Kamunen 2019) oder des Pausierens (Licoppe \& Tuncer 2014) einer der gleichzeitig relevanten Aktivitäten, wie auch das Wiederaufnehmen abgebrochener Aktivitäten (Sutinen 2014). Demnach können Interagierende den abgebrochenen Status einer Aktivität mithilfe von Accounts wie „wait/hang on“ (Keisanen, Rauniomaa \& Haddington 2014) oder „um“ (Sutinen 2014) markieren. Diese Betrachtung multipler Aktivitäten als eher nachfolgend koordinierte Ereignisse werden durch Studien kontrastiert, die solche Situationen fokussieren, in denen Interagierende in mehr als eine Aktivität gleichzeitig involviert sind (Raymond \& Lerner 2014; Deppermann 2014b; Hoey 2018a). Im Rahmen der multiactivity-Forschung werden abgebrochene bzw. pausierte multiactivities unter derselben Bezeichnung vereint wie simultan vollzogene Aktivitäten, obwohl die daran beteiligten Verfahren hinsichtlich ihrer Komplexität, Dauer und Teilnehmendenrahmung deutlich divergieren (vgl. Mondada 2014c). Dies ist zu betonen, da multiactivity häufig mit simultanem Handeln gleichgesetzt wird, während das Konzept zunächst nur beschreibt, dass für Interagierende mehrere Aktivitäten zur gleichen Zeit relevant werden. Dieser Konzeption folgend werden im Rahmen der vorliegenden Studie alle Aktivitäten, die für eine ${ }^{\star} n$ Teilnehmende ${ }^{\star} n$ in einer sozialen Situation gleichzeitig relevant werden als multiple Aktivitäten gefasst. Eine Präzisierung erfährt der Begriff durch die Unterscheidung in simultan und seriell koordinierte multiple Aktivitäten. Diese Unterteilung ist für die Fragestellung dieser Studie insofern sinnvoll, als Interagierende im Rahmen von simultan koordinierten multiplen Aktivitäten (wenn also tatsächlich mehrere Aktivitäten gleichzeitig vollzogen werden, z. B. in Form einer TANZENDEN-ERKLÄRUNG) komplexen Projekten nachgehen können, 
während bei seriell koordinierten multiplen Aktivitäten (z. B. TRINKEN und ERKLÄREN) die einzelnen Aktivitäten aufgrund möglicher struktureller Inkompatibilität um die verfügbaren multimodalen Ressourcen konkurrieren können und abwechselnd realisiert werden (vgl. Kap. 5). Zu den Studien, die sich simultan koordinierten multiplen Aktivitäten befassen, lassen sich die Arbeiten zum ERKLÄRENDEN-OPERIEREN in einem Operationssaal von Mondada (2011, 2014c), zUm ERZÄHLENDEN-ABKASSIEREN in einem Restaurant von Raymond \& Lerner (2014) oder zum INFORMIERENDEN-ANWEISEN in einer medizinischen Übung von Deppermann (2014b) zählen. Mit Ausnahme des letzten Beispiels handelt es sich bei den aufgeführten Multiaktivitäten allesamt um Koordinierungen von Ensemble- mit Einzelaktivitäten ${ }^{6}$. Simultan koordinierte multiple Aktivitäten aus mehreren ko-relevanten Ensembleaktivitäten, bilden hingegen in der multiactivity-Forschung bislang die Ausnahme (siehe Kapitel 6). Darüber hinaus sind Multiaktivitäten aus simultan koordinierten multiplen Einzelaktivitäten bislang noch nicht untersucht worden (siehe Kapitel 7). Bei der Betrachtung des aktuellen Forschungsstandes der multiactivity-Forschung fällt die Dominanz der sprachlichen Perspektive auf. Diese äußert sich darin, dass Koordinationsprozesse häufig lediglich für diejenigen Personen beschrieben werden, die sprechen und dabei etwas anderes tun (z. B. Kamunen 2020). Die kommunikative Arbeit, die nichtsprechende Interagierende dabei in Bezug auf die Koordination multipler Aktivitäten als doing being participant (Sacks 1992) leisten, rutscht bei dieser Betrachtungsweise aus dem Analysefokus. Dadurch kann fälschlicherweise der Eindruck entstehen, dass nur sprechende Interagierende in Multiaktivitäten involviert wären. Wenn vorrangig verbale Beteiligungsweisen betrachtet werden, stellt sich für Interaktionsforschende die Frage nicht, welche Rolle die strukturelle Kompatibilität für den simultanen Vollzug multipler Aktivitäten in Bezug auf die Koordination ko-relevanter Aktivitäten spielt. Denn mit einer sprachzentrierten Perspektive interessiert lediglich der Umstand, ob eine andere Aktivität das Sprechen behindert oder nicht. Strukturelle Inkompatibilitäten nicht-sprachlicher Beteiligungsweisen werden infolgedessen ignoriert und die Verfahren, mit denen die Interagierenden einer strukturellen Inkompatibilität der Beteiligungsweisen begegnen, können nicht erfasst werden. Um diesem einseitigen sprachzentrierten Ansatz entgegenzuwirken und eine kommunikationswissenschaftliche Perspektive einzunehmen, wird das Phänomen gleichzeitiger

6 Wie in 2.3.2 ausführlicher dargestellt wird, handelt es sich bei einer Ensembleaktivität um eine intersubjektiv hergestellte Aktivität eines Interaktionsensembles. Einzelaktivitäten werden nicht intersubjektiv ausgehandelt und damit häufig von einzelnen Personen innerhalb des Interaktionsensembles realisiert. Bei beiden Aktivitätsformen handelt es sich um Teilnehmendenkategorien, die im Rahmen von sozialen Situationen (Goffman (1963) auftreten können. 
Relevanz multipler Aktivitäten in einer Interaktion in dieser Studie vom Interaktionsensemble aus betrachtet. Ein Interaktionsensemble umfasst „personelle, räumliche und thematisch-pragmatische Konstellationen als von den Beteiligten gemeinsam konstituierte Form von Kooperation sowie interaktiver Beteiligung und Bezogenheit“ (Schmitt 2012: 79) in Situationen gegenseitiger Wahrnehmung. In Multiaktivitäten involvierte Interagierende müssen durch intrapersonelle Koordination (Deppermann \& Schmitt 2007; Deppermann 2014b) mindestens zwei Aktivitäten „ko-relevant“ (Haddington et al. 2014: 3) halten, während sie im Rahmen ihrer interpersonellen Koordinierung (Deppermann \& Schmitt 2007) die Beteiligungsweisen an den multiplen Aktivitäten mit den anderen Teilnehmenden der sozialen Situation ausrichten müssen (Deppermann 2014b: 249). Für die Ko-Interagierenden ergibt sich daraus die Anforderung, die Multiaktivität mit deren unterschiedlichen temporalen Logiken der Teil-Aktivitäten zu monitoren und die eigenen Beteiligungsweisen darauf auszurichten. Das bedeutet, dass das Phänomen der Gleichzeitigkeit in dieser Arbeit nicht nur unter intrapersonell koordinativen Gesichtspunkten betrachtet wird, sondern auch immer in Bezug auf die interpersonelle Koordination jener Beteiligter, die ihre Beteiligungsweisen auf mehrere potentiell gleichzeitig relevante Aktivitäten ausrichten. So werden die Analysen in den Kapiteln 5-7 zeigen, dass den sprachlichen Ressourcen zwar durchaus eine wichtige Bedeutung zur Herstellung von Intersubjektivität zukommen kann, die simultane Koordination von multiplen Aktivitäten aber auch gänzlich sprachfrei ablaufen kann. Häufig werden in der multiactivity-Forschung Einzelaktivitäten (wenn überhaupt) als sprachfrei und ausschließlich Ensembleaktivitäten als sprachlich organisiert beschrieben. Das Auftreten sprachfreier Ensembleaktivitäten wird zwar stellenweise angedeutet (z.B. in Nishizaka 2014), bleibt aber hinsichtlich einer koordinativen Integration in die laufende Multiaktivität unterentwickelt. Deshalb wird in der multiactivity-Forschung zwar durchaus häufig beschrieben, dass Teilnehmende Einzel- zugunsten von Ensembleaktivitäten abbrechen, die Beobachtung des diametralen Falls findet sich jedoch nur vereinzelt (z. B. in Oloff in prep.). Um diesem Desiderat zu begegnen, werden in den Analysekapiteln 5-7 die Koordinationspraktiken bei der Bearbeitung gleichzeitiger Relevanz multipler Aktivitäten unabhängig von ihren sprachlichen Beteiligungsweisen im Rahmen der jeweils zugrundeliegenden Fallkollektionen analysiert. Dafür werden bei den Analysen auch jene Verfahren aufgegriffen, die von der multiactivity-Forschung bereits beschrieben werden (wie das Abbrechen oder das Pausieren), um sie allerdings zum einen für Einzel- und Ensembleaktivitäten sowie zum anderen - und das ist neu in der multiactivity-Forschung -hinsichtlich ihrer strukturellen (In)Kompatibilität systematisch zu variieren und auf die Koordination multipler Aktivitäten in Interaktionen anzuwenden. 


\subsection{Zeitlichkeit in der Interaktionsforschung}

Interaktionen sind Prozesse, in denen Phänomene von sehr kurzer Dauer relevant werden (z. B. 30 ms bei Blickwechseln zwischen Adressat*innen, vgl. Holmqvist et al. 2011) oder längere Zeitabschnitte umfassen können (z. B. mehrstündiges Diskutieren einer Textfassung während einer Theaterprobe). Zeit und Zeitlichkeit sind demnach von essentieller Bedeutung für die Organisation sozialer Situationen. Umso überraschender ist es, dass erst Ende der 1990er/Anfang der 2000er Jahre damit begonnen wurde, „die Zeitlichkeit der mündlichen Sprache ernst zu nehmen“ (Auer 2000) und dies explizit als Untersuchungsgegenstand der Interaktionsforschung zu benennen. Denn anders als Schrift ist gesprochene Sprache ein flüchtiges Zeichensystem, das im Moment der Realisierung vergeht. Darüber hinaus sind satzhafte Äußerungseinheiten nicht sofort vollständig wahrnehmbar (im Gegensatz zu beispielsweise einer Seite geschriebenem Text), sondern bedürfen einer Nacheinanderproduktion der einzelnen Äußerungsbestandteile (Lexeme, Partikeln, Pausen, usw.). In der linguistischen Interaktionsforschung („Interaktionale Linguistik“, Couper-Kuhlen \& Selting 2018) wurde diese Prozesshaftigkeit von gesprochener Sprache unter anderem als Online-Syntax (Auer 2000, 2007a) konzeptualisiert oder in Hinblick auf Inkrementalität und Emergenz (Günthner 2007, 2011a) untersucht. Bezugnehmend auf die Studien zu nonverbaler Kommunikation in der psychologischen Forschung der 1970er Jahre (z. B. zu Gesten: Kendon 1972; zu Mimik: Ekman \& Friesen 1978) und dem darauf aufbauenden multimodalen Zugang (vgl. Mondada 2019a) erfolgte im Anschluss eine Auseinandersetzung mit Zeitlichkeit in (multimodalen) Interaktionen (Deppermann \& Günthner 2015; Deppermann \& Streeck 2018). Im Zuge dessen rückten die temporalen Logiken unterschiedlicher Vorgänge (z. B. Sprechen und Tanzen, Keevallik 2015), die Prospektivität deiktischer Projektionen (Stukenbrock 2018b) oder die Retrospektivät bei verzögerten Abschlüssen (Oloff 2018) in den Forschungsfokus. Zeitlichkeit in Interaktionen, das wird in diesen Arbeiten deutlich, kann mit Deppermann \& Streeck (2018: 4) in drei Bereiche eingeteilt werden:

a) Zeitlichkeit als Dauer: z. B. Sprechpausen, gedehnte Silben, Bewegungsphasen einer Geste und ganze Handlungssequenzen als „Zeit-Objekte“

b) Zeitlichkeit als Bezugsrahmen: z. B. retrospektive und prospektive Perspektiven der Teilnehmenden auf interaktionale Ereignisse

c) Zeitlichkeit als Timing: z. B. zeitliche Koordinierung verschiedener Phänomene wie die Verknüpfung von Gestik und Sprache

Im weiteren Verlauf dieser Studie werden vorrangig Aspekte des Timings bei der interaktionalen Bearbeitung gleichzeitig auftretender Relevantsetzungen in Handlungssequenzen („Aktivitäten“) untersucht. Daher soll zunächst dargestellt 
werden, wie Gleichzeitigkeit in Arbeiten der Interaktionsforschung bislang konzeptualisiert und analytisch bearbeitet worden ist. Dazu wird der forschungshistorischen Chronologie folgend zunächst die sequentielle Gleichzeitigkeit (2.2.1) und anschließend die multimodale Simultanität (2.2.2) betrachtet, um daraus den analytischen Umgang mit Gleichzeitigkeit im Rahmen dieser Studie abzuleiten.

\subsubsection{Sequentielle Gleichzeitigkeit}

Wenn Menschen miteinander reden, wechseln sie sich dabei ab und produzieren nur selten gleichzeitig Sprachbeiträge. Mit dieser zunächst trivial wirkenden Beobachtung beschäftigt sich der für die Konversationsanalyse zentrale Aufsatz „A simplest systematics for the organization of turn-taking for conversation“ (Sacks, Schegloff \& Jefferson 1974), indem er Antworten auf die Fragen liefert: Aus welchen Gründen kommt es in Face-to-Face-Interaktionen vergleichsweise selten zu Überlappungen der Interaktionsbeiträge? Woher wissen Interagierende, wann der Sprachbeitrag („Turn“) des`der vorherigen Sprecher^in abgeschlossen ist? Die zentrale Erkenntnis des Aufsatzes ist, dass Interagierende die Sprachbeiträge der anderen auf sogenannte übergaberelevante Stellen („transition relevance places/TRPs“) hin überprüfen. Damit entsteht ein hocheffizientes System des Sprecher^innen-Wechsels („Turn-Taking“), bei dem weniger als 5\% der Sprechbeiträge einander überlappen (Levinson 2016: 6). Wie Levinson \& Torreira (2015) zeigen konnten, beträgt die Dauer zwischen zwei Turns durchschnittlich ca. 200 ms. Das ist insofern interessant, als die kognitiven Prozesse der Sprachproduktion etwa 600 ms benötigen. Daraus lässt sich schlussfolgern, dass die Sprachproduktionsplanung im Durchschnitt bereits ca. 400 ms vor dem Ende des Turns des $^{\star}$ der vorherigen Sprecher*in einsetzen muss. Um also Sequenzen aufeinander bezogener und lokal kohärenter Sprechbeiträge herstellen zu können, müssen Interagierende einander zuhören und gleichzeitig ihre eigene Sprachproduktion beginnen. Die Verfahren zur Herstellung solcher Sequenzen aufeinander bezogener Interaktionsbeiträge stehen seit jeher im Forschungsfokus der Konversationsanalyse. Damit bildet die Auseinandersetzung mit (fehlender) Gleichzeitigkeit zwar einen Ausgangspunkt für die Forschungslinie der Konversationsanalyse, spielt jedoch in ihrer weiteren Entwicklung eine eher untergeordnete Rolle. Denn das Verständnis von Interaktionen im konversationsanalytischen Sinne ist das der sequentiellen Organisiertheit: „they talk singly - that is, one at a time“ (Schegloff 2007: 1).

Gleichzeitigkeit rückt nur dann in den (an sprachlichen Phänomenen interessierten) Analysefokus, wenn die Grenzen der Sequentialität von den Interagierenden bearbeitet werden, z. B. wenn sie trotz Turn-Taking-Mechanismus einan- 
der verbal überlappen. Überlappungen („overlaps“) gehören zu den am frühesten untersuchten gleichzeitigen Phänomenen der Konversationsanalyse (Jefferson 1973, 1986; Schegloff 2000b). In den Arbeiten zu diesem Phänomen wird herausgearbeitet, wie Interagierende die Abweichung der Maxime one at a time bearbeiten (z. B. lauter/schneller sprechen als der ${ }^{\star}$ die Konkurrent*in), um danach wieder zum regulären Turn-Taking übergehen zu können. Überlappungen als gleichzeitiges Handeln zweier Beteiligter werden damit in diesen Studien als bearbeitungsbedürftige, interaktionale Probleme der Teilnehmenden verstanden.

Ein weiteres Phänomen der Gleichzeitigkeit, das mit einer sequentiellen Perspektive auf Interaktionen beschrieben werden kann, ist das der Nebenorganisation mehrerer Sequenzen. In ihrem Beitrag „Side Sequences“ beschreibt Jefferson (1972) Gesprächsstränge, die zugunsten anderer Gesprächsstränge unterbrochen werden. Die Besonderheit einer solchen unterbrechenden Nebensequenz ist, dass sie den Gesprächsstrang der Hauptsequenz nicht beendet, sondern lediglich pausiert. Damit sind für die Teilnehmenden zum Zeitpunkt der Herstellung einer solchen Nebensequenz zwei Sequenzen gleichzeitig relevant: die aktuelle Nebensequenz und die pausierte Hauptsequenz, die fortgesetzt wird, sobald die Nebensequenz ihren Abschluss gefunden hat. Werden Gesprächsstränge nicht unterbrochen, sondern gleichzeitig und einander überlappend realisiert, kann dies im Rahmen eines Prozesses stattfinden, den Egbert (1993) „Schisming“ nennt. Dabei bricht ein Gespräch in mehrere Stränge auf, die jeweils eigene sequentielle Strukturen ausbilden. Interagierende stellen das Schisming her, indem sie eine neue Handlung oder ein neues Thema relevant setzen. Sie lösen das gleichzeitige Nebeneinander der Sequenzen dadurch auf, dass sie eine der Sequenzen abschließen und sich visuell auf die Teilnehmendenrahmung der anderen Sequenz orientieren, an der sie sich auf diese Weise wieder beteiligen. Ein ähnliches Verständnis von Gleichzeitigkeit in der Sequentialität findet sich ins Schegloffs Beitrag „Body Torque“ (Schegloff 1998), in dem er die Drehung des Oberkörpers als interaktionales Verfahren zur Organisation von Beteiligung an mehreren Interaktionssträngen beschreibt. Body Torque als Verfahren ist in zweierlei Hinsicht von besonderer Bedeutung für die Konzeptualisierung von (sequentieller) Gleichzeitigkeit in Interaktionen. Zum Ersten zeigt sich, dass Gleichzeitigkeit für die Teilnehmenden kein von der (interaktionalen) Norm abweichendes Problem per se darstellen muss (wie z. B. Überlappungen). Vielmehr nutzen Interagierende die Drehung des Oberkörpers, um einander ihre Involvierung in den laufenden Interaktionsstrang anzuzeigen. Damit wird deutlich, dass Gleichzeitigkeit einen konstitutiven Bestandteil in sozialen Situationen darstellen kann. Sequentielle Gleichzeitigkeit kann daher als Nacheinander-Bearbeiten von relevant gehaltenen Sequenzen in Interaktionen verstanden werden. Zum Zweiten nimmt Schegloff neben Goodwin (z. B. Goodwin 1980a) mit der Untersuchung der 
Körperhaltung bzw. Blick als interaktionale Ressourcen den multimodalen Turn in der Konversationsanalyse der 2000er Jahre vorweg. Aus dieser Perspektive heraus ist es möglich, Gleichzeitigkeit nicht nur als Relevanthalten von Sequenzen zu verstehen, sondern darüber hinaus auch als Simultanität körperlicher Verfahren $\mathrm{zu}$ beschreiben.

\subsubsection{Multimodale Simultanität}

Wenn Menschen in Face-to-Face-Interaktionen miteinander sprechen, schauen sie einander währenddessen an, gestikulieren und verwenden Gesichtsausdrücke. Dies geschieht zu einem großen Teil nicht nacheinander (sequentiell), sondern simultan: eine Geste kann zeitgleich mit einem Sprachbeitrag erfolgen, parallel zu einem Gesichtsausdruck ausgeführt und während einer bestimmten Körperhaltung vollzogen werden. Das gleichzeitige Vorhandensein verschiedener Ausdrucksressourcen wird in der Interaktionsforschung als Multimodalität bezeichnet und konstituiert das menschliche Miteinander. Jede ${ }^{7}$ Face-to-FaceInteraktion ist demnach multimodal organisiert.

Die Betrachtung menschlicher Interaktionen als multimodale Kommunikationsprozesse ist wissenschaftshistorisch ein sehr junger Ansatz, der besonders ab den 2000er Jahren an Popularität gewonnen hat. Die Grundlagen dieser Forschungsrichtung wurden aber bereits deutlich früher gelegt und reichen bis zu den Arbeiten von beispielsweise Hall (1963), Birdwhistell (1970) und Argyle (1975) zurück, die unter anderem Ressourcen der Körperpositur, Proxemik und Gestik in Relation zu sprachlichen Einheiten betrachten. Von der interaktionsanalytisch orientierten Forschungsgemeinschaft besonders häufig rezipiert wurden bzw. werden die frühen interaktionistischen Arbeiten Erving Goffmans (1963, 1979, 1981). In seinen für moderne interaktionsanalytische Forschungen noch immer bedeutsamen Schriften spielt Multimodalität für die Beschreibung alltäglicher Interaktionen bereits eine wichtige Rolle. Goffman geht in seinen sich vorwiegend auf Beobachtungen stützenden Arbeiten von einem gleichberechtigten Nebenund Miteinander verschiedener Ressourcen aus - eine Annahme, die bei empirisch arbeitenden Wissenschaftlern zunächst zugunsten sprachlicher Ressourcen

\footnotetext{
7 Selbst Gespräche am Telefon fußen zunächst auf der multimodalen Verfasstheit von Interaktionen: Teilnehmende einer solchen Situation gestikulieren, produzieren Gesichtsausdrücke und agieren mit dem Telefon als Objekt in solcher Weise, dass die Interaktion aufrechterhalten bleiben kann. Dass dem Gegenüber diese Ressourcen jedoch nicht in unmittelbar wahrnehmbarer Form zugänglich sind, ist der Grund dafür, dass die frühe konversationsanalytische Forschung sich intensiv mit den sprachlichen Praktiken in Telefongesprächen auseinandergesetzt hat.
} 
in den Hintergrund gerückt ist und erst seit wenigen Jahren wieder verstärkt propagiert wird. Das Vorhandensein von Sprache ist in den Arbeiten von Goffman kein unabdingbares Kriterium für Interaktionen in sozialen Situationen. Auch Kleidung, Verhalten, Bewegung, Position, Lautstärke, physische Gesten (z. B. Winken oder Grüßen), Schmuck und emotionaler Ausdruck (Goffman 1963: 33) stellen bei ihm wichtige und für Analysen zu berücksichtigende Ressourcen dar. Das geht soweit, dass Kommunikation für Goffman auch ohne Sprache funktionieren kann: „Although an individual can stop talking, he cannot stop communicating through body idiom“ (Goffman 1963: 35). Gerade diese konstante Simultanität der Ausdrucksressourcen macht die Erforschung multimodaler Interaktionen schwierig und aufwendig. Die Körper aller Interagierender sind ständig in Bewegung, weshalb sich frühe Analysen der Multimodalitätsforschung auf einzelne Ressourcen beschränkt haben und jeweils herausarbeiteten, in welcher eng verzahnten Relation die jeweilige Ressource mit sprachlichen Äußerungen steht.

Charles und Marjorie Goodwin (1979, 1980a; 1980b) zeigen an einem Korpus von 50 Stunden Videoaufzeichnungen von Tischinteraktionen in einer Reihe von Arbeiten den Einfluss des Blickkontakts auf die Sprachproduktion, zum Beispiel in Bezug auf Pausen oder Reformulierungen. Adam Kendon (1980, 2004) untersucht den Zusammenhang zwischen Gestikulation und Sprache und arbeitet heraus, dass Körper- und Sprachaktivitäten untrennbar miteinander verbunden sind: „It is as if the process of utterance has two channels of output into behavior: one by way of speech, the other by way of bodily movement“ (Kendon 1980: 218). Er beschreibt Experimente, in denen Probanden die Aufgabe gestellt wurde zu reden, ohne zu Gestikulieren oder Mimik einzusetzen. Es kam in allen Fällen zu Problemen der Sprachproduktion, was Kendon als Beweis für die Untrennbarkeit der sprachlichen und gestikulatorischen Ressourcen wertet. Auf Kendon aufbauend und ihn erweiternd kommt auch McNeill (1992, 2005) zu dem Schluss, dass Sprache und Gesten Teile desselben Systems seien, allerdings aufgrund ihrer unterschiedlichen spezifischen semiotischen Kapazität andere Funktionen übernehmen, zum Beispiel ein verbal nur vage beschriebenes Objekt mithilfe einer ikonischen Geste konkreter darzustellen. Besonders die McNeill'schen Daten zeigen ein Spezifikum der frühen Multimodalitätsforschung: Seine Analysen beruhen auf Videoaufzeichnungen einzelner Personen in experimentellen Settings ohne Betrachtung des interaktionalen Potentials von Gesten in dyadischenoder Mehrpersonen-Gesprächen. Während Videocamcorder in den 1980er Jahren vor allem als Teil psychologischer und sozialpsychologischer Untersuchungen in experimentellen Umgebungen eingesetzt wurden, bleibt die Arbeit an authentischen Daten und damit die Analyse menschlicher Interaktion vergleichsweise selten (vgl. Heath 1986: 3). Es fehlt nicht nur an Material, sondern auch an einer entsprechenden Methodologie zum Umgang mit den großen Daten- und Infor- 
mationsmengen, mit denen sich Multimodalitätsforschende auseinandersetzen müssen.

Die ab den 2000er Jahren einsetzende „,visuelle Revolution“ (Schmitt 2005: 23) geht mit einer Weiterentwicklung der bestehenden ethnomethodologisch-konversantionsanalytischen Methodologie einher. Über 20 Jahre nach den ersten, auf Grundlage von audiovisuellem Datenmaterial durchgeführten, Interaktionsanalysen sind die Erkenntnisse über Sprache, Interaktion und Kognition so weit, dass die Integration körperlicher Ressourcen in konversationsanalytische Arbeiten den nächsten logischen Schritt darstellt. Nicht nur international, auch innerhalb des deutschsprachigen Raumes erschienen und erscheinen immer mehr interaktionslinguistische Arbeiten mit primär multimodalem Ansatz: Dausendschön-Gay, Gülich \& Krafft (2015), Deppermann \& Schmitt (2007), Fricke (2012), Schmitt (2005), Schmitt \& Knöbl (2014), um nur einige zu nennen. In diesen Arbeiten besteht Konsens darüber, dass alle Modalitäten für Interagierende gleich relevant sein können und daher in den Analysen keine als den anderen automatisch übergeordnet behandelt werden darf - außer eine bestimmte Ressource wird von den Teilnehmenden explizit relevant gesetzt, z. B. eine Geste bei Nicht-Nachkommen einer Aufforderungshandlung. Die dabei eingesetzten Modalitäten kommen in menschlicher Interaktion weder einzeln vor, noch werden sie von Interagierenden separat wahrgenommen ${ }^{8}$. Die Gesamtheit aller wahrnehmbaren multimodalen Ressourcen eines ${ }^{\star}$ r Teilnehmenden in einer Situation bilden eine Gestalt. Das Gestalt-Konzept kommt der holistischen Perspektive auf Multimodalität in der Interaktionsforschung sehr nahe und bildet den Kern der Gestalttheorie. Diese Perzeptionstheorie wurde in den 1920er und 1930er Jahren von einer Reihe von Psychologen (Wertheimer 1923, 1925; Köhler 1928; Koffka 1928, 2013 [1935]) entwickelt, die zu erklären versuchen, wie und warum individuelle Elemente in der Wahrnehmung zu Clustern zusammengefasst werden. Diese gruppierten Einzelelemente konzeptualisieren Gestaltforscher als Gestalten, die im sogenannten Wahrnehmungsraum der Sinnesorgane liegen. Im Laufe der Zeit wurden 13 Prinzipien herausgearbeitet, nach denen Menschen individuelle Einheiten zu Gestal-

\footnotetext{
8 Darüber hinaus sind viele Sinnesorgane des Menschen gleichzeitig bei der Perzeption der Modalitäten beteiligt: Sprache kann in Form der Lippenbewegung gesehen (unter bestimmten Umständen auch gerochen und gefühlt), Bewegung als Kleidungsrascheln oder als Fußschritte gehört und Gestik als Berührung gespürt werden. Vermutlich ist der Geschmackssinn der einzige, der sich nicht zur Wahrnehmung von menschlicher Kommunikation eignet. Siehe für die synästhetische Verbindungen mehrerer Modalitäten auch Tilot et al. (2018). Siehe Speed \& Majid (2018) für den (nicht-existenten) Einfluss von Gerüchen auf die kognitive Verarbeitung von Lexemen.
} 
ten zusammenfügen. ${ }^{9}$ Das zentrale Prinzip ist dabei das der Übersummativität. Diesem zufolge ist das Ganze (in der Wahrnehmung) mehr als die Summe seiner Einzelteile. Dieses Prinzip auf multimodale Untersuchungen zu übertragen liegt nahe und ermöglicht interaktionale Displays als multimodale Gestalten simultan relevanter Ressourcen zu konzeptualisieren, die sich aus dem situativen Tun der Beteiligten ergeben. Aufgrund der Fokussierung auf sprachliche Interaktionsbeiträge wurde das Gestalt-Konzept bislang oft nur auf isolierte Ressourcen, wie Prosodie (Auer \& Couper-Kuhlen 1994; Couper-Kuhlen 2009; Günthner 2018) oder Gestik (Streeck 1993) im Verhältnis zu verbalen Einheiten angewendet. Krafft \& Dausendschön-Gay (2003; vgl. auch Dausendschön-Gay \& Krafft 2002) erweitern diese Perspektive um ihr holistisches Konzept der kommunikativen Gestalten und zeigen in ihren Analysen, dass Teilnehmende einzelne Äußerungen durch ihre Gestalthaftigkeit als multimodale Einheiten behandeln. Mondada (2014a: 136) führt diesen Gedanken konsequent weiter, indem sie alle Interaktionsbeiträge als „complex multimodal gestalts“ beschreibt. Demnach sind Zeigegesten nicht nur als Arm- und Handbewegungen zu beschreiben, sondern als Bewegung des gesamten Körpers mit allen multimodalen Ressourcen (inkl. Positur, Blick, Mimik, usw.) in ihren sequentiellen Umgebungen.

Mit dem Verständnis einer simultanen Relevanz multimodaler Ressourcen als multimodale Gestalt wird ein als „Primat der Verbalität“ (Schmitt \& Knöbl 2013: 244) bezeichneter analytischer Fokus vermieden. Darüber hinaus wirkt diese Konzeption von multimodaler Simultanität einer isolierten Betrachtung einzelner körperlicher Ressourcen entgegen wie zum Beispiel der häufig von interaktionalen Prozessen losgelösten Betrachtung der Gesten in der Gestenforschung (Müller 1998; McNeill 2014; Bressem 2007). Die Aufgabe multimodaler Interaktionsanalysen ist es nun, unter Berücksichtigung und Reflektion der Erkenntnisse bezüglich der internen Logiken der einzelnen Modalitäten ${ }^{10}$, zu rekonstruieren, wie diese Modalitäten simultan zueinander als gleichberechtigte Ressourcen in Interaktionen von Interagierenden benutzt werden, um bestimmte Interaktionsaufgaben zu bearbeiten, z. B. im Rahmen der Koordination multipler Aktivitäten.

Mit einer konsequent multimodalen Perspektive rückt darüber hinaus die Organisation der multimodalen Ressourcen als inter- und intrapersonelle Koordinationsleistung (vgl. Deppermann \& Schmitt 2007: 32-34) in den Forschungsfokus.

9 Einen Überblick über diese Prinzipien findet sich bei Chang (2007).

10 Beispielsweise die sequentielle Organisation von Sprache bei Sacks, Schegloff \& Jefferson (1974); die Phasen des Gestikulierens wie die Gestenvorbereitung (preparation), Gestenausführung (stroke) und Gestenrückführung (recovery) bei Kendon (1980); oder die Simultanität des körperlichen Ausdrucks bei Deppermann \& Schmitt (2007). 
Unter interpersoneller Koordination werden jene multimodalen Verhaltensweisen gefasst, mit denen Interagierende sich mit anderen Beteiligten interaktiv abstimmen. Darunter fallen alle sequentiellen Verfahren der Konversationsanalyse: Wer handelt wie bezogen auf wen? Wie zeigen Interagierende ihr Verständnis der vorherigen Interaktionsbeiträge an? Neu in den Analysefokus rückt mit dem Konzept der Koordination die Selbstorganisation der multimodalen Ausdrucksmodalitäten als intrapersonelle Koordination: Wann erreicht die Geste ihren Höhepunkt (Stroke bzw. Peak, Kendon 1980)? Wie stellen Interagierende die Simultanität ihrer multimodalen Ressourcen her? Deutlich wird dabei, dass in Interaktionen immer sowohl inter- als auch intrapersonelle Koordinierung als adaptiver Prozess betrieben wird. Das bedeutet, dass die interpersonelle Koordination Einfluss auf die intrapersonelle Koordination haben kann und vice versa (z. B. wenn bei einer „delay-Organisation“ die eigene Zeigegeste aufrechterhalten bleibt, weil der^die Adressierte sich körperlich noch nicht auf das Zeigeziel ausgerichtet hat, vgl. Deppermann \& Schmitt 2007: 28). Mit dem Konzept der Koordination wird es möglich, die Achse der sequentiellen Gleichzeitigkeit und die Achse der multimodalen Simultanität aufeinander $\mathrm{zu}$ beziehen und damit die wechselseitigen Prozesse der multimodalen Selbstorganisation und der Abstimmung mit anderen Interagierenden bei der Organisation multipler Aktivitäten analytisch zu fassen. Eine besondere Form der Koordinierung, auf die näher in Kapitel 6 eingegangen wird, ist die Synchronisierung. Bei diesem Koordinierungsprozess werden multimodale Ressourcen und Beteiligungsweisen miteinander aligniert. Wie Breyer \& Pfänder (2017: 12-14) beschreiben, geschieht diese Synchronisierung sowohl im Rahmen von intrapersoneller Koordinierung (Selbstsynchronisierung) als auch als Prozess von interpersoneller Synchronisierung, wobei der rhythmischen Organisation der Interaktionsbeiträge eine zentrale Rolle zukommt. Interaktionale Perspektiven auf Synchronisierung gibt es bislang kaum. Somit nimmt der Sammelband von Thiemo Breyer, Michael B. Buchholz, Andreas Hamburger, Stefan Pfänder, Elke Schumann (2017) zur Synchronisierung eine zentrale Stellung in diesem Feld ein, wenngleich dort die Synchronisierung von multiplen Aktivitäten fehlt. Dieses Desiderat wird mit der vorliegenden Arbeit eingelöst.

Diese Prozesse der Koordinierung stellen komplexe Daueranforderungen an die Teilnehmenden, bei denen alle Interagierende mehr oder minder intensiven gleichzeitig relevanten Interaktionsaufgaben (simultan die anderen beobachten und selbst agieren) ausgesetzt sind. Aber so wie bei der sequentiellen Gleichzeitigkeit immer nur eine Sequenz bearbeitet wird, beschreibt die multimodale Simultanität zunächst nur die gleichzeitige Relevanz bestimmter Ausdrucksressourcen innerhalb einer Aktivität. Ziel dieser Studie ist die Analyse der Verfahren, mit denen Teilnehmende die gleichzeitige Relevanz mehrerer Aktivitäten bearbeiten. Dass dabei nicht pro Aktivität separate Koordinierungsprozesse 
angenommen werden können, die in der Interaktion addiert werden (die Anzahl der Modalitäten ist schließlich begrenzt), wird bei der folgenden Diskussion des Aktivitätskonzepts in der Konversationsanalyse (2.3) deutlich werden.

\subsection{Aktivitäten als interaktionale Kategorien der Partizipation}

Die Aktivitäten, die Menschen in Interaktionssituationen vollziehen, sind vielfältig: Erzählen, Berichten, Essen, Kleidung ABlegen usw. Für die Interagierenden sind dies die Verfahren, mit denen sie ihren Alltag organisieren. Den Beteiligten an den Interaktionen scheint es mit Leichtigkeit zu gelingen, die unterschiedlichen Aktivitäten zu verstehen und darauf zu reagieren. Bei der Erforschung dieser Aktivitäten und ihrer Koordinationsverfahren bei gleichzeitiger Relevanz stellt sich hingegen die Herausforderung, die Analysekategorie Aktivität adäquat zu erkennen, zu beschreiben und von verwandten Begriffen wie Handlungen und Praktiken abzugrenzen. Besonders letzteres gestaltet sich mitunter schwierig, weil sich die englischen und die deutschen Begriffe activity/ Aktivität, action/Handlung und practice/Praktik nicht gänzlich semantisch kongruent zueinander verhalten. In diesem Abschnitt soll daher eine Abgrenzung versucht und eine Begriffsdifferenzierung des Aktivitätsbegriffs für die deutsche Interaktionsforschung vorgeschlagen werden. Im Kern wird für folgende hierarchische und in Bezug auf ihre granulare Auflösung differenzierte Beziehung der genannten Begriffe argumentiert: Eine Aktivität bildet einen Orientierungsrahmen für Handlungen, die ihrerseits durch Praktiken realisiert werden.

Obwohl sich dieses Verständnis von Aktivitäten in einer Reihe von konversationsanalytischen Arbeiten wiederfindet (siehe 2.3.1), drückt sich die bereits angedeutete Unschärfe der Kategorie Aktivität innerhalb der Interaktionsforschung in unterschiedlichen und zum Teil gegenläufigen konzeptionellen Ausrichtungen aus. Für die Untersuchung der Organisation mehrerer Aktivitäten in einer sozialen Situation ${ }^{11}$ (folgend multiple Aktivitäten) ist allerdings ein schärferer Begriff nötig, weshalb zunächst die ethnomethodologische Basis der Kategorie Aktivität skizziert werden soll, um anschließend den für diese Studie geltenden konversationsanalytischen Aktivitätsbegriff (2.3.1) und die sich daraus ergebende Unterscheidung in Einzel- und Ensembleaktivitäten (2.3.2) vorzustellen.

11 „I would define a social situation as an environment of mutual monitoring possibilities, anywhere within which an individual will find himself accessible to the naked senses of all others who are 'present,' and similarly find them accessible to him. According to this definition, a social situation arises whenever two or more individuals find themselves in one another's immediate presence, and it lasts until the next-to-last person leave“ (Goffman 1964: 135). 
Die Auseinandersetzung mit der analytisch schwierig $\mathrm{zu}$ fassenden Kategorie Aktivität beginnt mit der Feststellung, dass es sich dabei nicht nur um ein methodologisch-konzeptuelles Problem, sondern auch um ein ethnomethodologisches Problem handelt: Die Interagierenden stehen wie die Analysierenden vor der Herausforderung, dem körperlichen Tun der anderen Interagierenden einen Sinn beizumessen und das Tun der anderen als Bestandteil einer bestimmten Aktivität zu interpretieren. Wie die Analysierenden können sie dabei nur das nutzen, was die anderen Beteiligten ihnen mittels multimodaler Ressourcen als Interpretationshinweise anbieten bzw. relevant setzen. Das bedarf einer konstanten Beobachtung der Ko-Interagierenden, um deren Aktivitäten wahrzunehmen und auf sie zu reagieren. Eine innerhalb einer sozialen Situation hervorgebrachte Aktivität kann demnach als eine Kategorie der Partizipation (z. B. Goodwin 2007) am interaktionalen Geschehen verstanden werden. Zentral bei diesem Verständnis von sozialen Aktivitäten als Partizipationskategorien ist die Organisation von Aktivitäten als „endlessly contingent manifestations of real-worldly conduct“ (Maynard \& Clayman 1991: 390). Dies ist insofern die wichtigste Voraussetzung dafür, dass Aktivitäten (bzw. soziale Handlungen im Rahmen von Aktivitäten) accountable (Garfinkel 1967) gemacht werden können, als Beteiligte nur dann auf die sozialen Handlungen einer Aktivität regieren können, wenn sie diese wahrnehmen ${ }^{12}$ können (vom Lehn 2019). Somit sind rein mentale Vorgänge („mental actions”, Deppermann \& Streeck 2018: 7) wie beispielsweise Kopfrechnen, Tagträumen oder sich an ein Rezept erinnern analytisch zunächst nicht als Bestandteile von Aktivitäten erfassbar. Interagierende haben allerdings die Möglichkeit, diese Vorgänge unter Rückgriff auf multimodale Ressourcen als wahrnehmbare Aktivitäten zu gestalten. Da Aktivitäten erst in ihrer situativen Gestaltung hervorgebracht werden, sind sie keine absoluten Kategorien. Vielmehr kann ein Verhalten, das innerhalb einer sozialen Situation als eine Aktivität behandelt wurde, für die Teilnehmenden in einer anderen Situation eine ganz andere Aktivität darstellen. Aktivitäten stehen demnach immer in relationalem Verhältnis zur kontextuellen Konfiguration und können in ihrer situativen, lokalen Verortung selbsterklärend ${ }^{13}$ sein (Atkinson 1988: 446).

12 Im phänomenologischen Sinne von Merleau-Ponty (2016 [1946]: 83): „sich etwas mit Hilfe des Leibes zu vergegenwärtigen“.

13 Aktivitäten sind selbsterklärend für die Teilnehmenden des Settings, aber nicht zwangsläufig auch für Beobachtende oder Analysierende. Für eine Analyse von Aktivitäten im Theater ist (ethnographisches) Wissen zwingende Voraussetzung (vgl. Kap. 4 dieser Studie). Dies schließt die kulturelle Gebundenheit von Aktivitäten ein: Angehörige verschiedener Kulturen könnten von derselben Aktivität unterschiedliche Verständnisse haben, die sie in Interaktionen erst aushandeln müssen (vgl. Hubrich 2015: 41). 
Anhand der Aushandlungsprozesse von Aktivitätsübergängen (z. B. Deppermann, Schmitt \& Mondada 2010, Keevallik 2010b) lässt sich darüber hinaus erkennen, dass Aktivitäten für die Teilnehmenden keine isolierten interaktionalen Einheiten darstellen, sondern von vorherigen und nachfolgenden Aktivitäten eingerahmt werden. Damit sind Aktivitäten nicht nur Hervorbringungen menschlicher Interaktion, sondern erzeugen als Aktivitätssequenzen die Ordnung des sozialen Lebens (Atkinson 1988: 447). Diese Aktivitätssequenzen werden in komplexen Raum-Zeit-Beziehungen organisiert, wie beispielsweise Lynch (1985) anhand seiner Untersuchung von wissenschaftlichen Praktiken in einem neurowissenschaftlichem Labor zeigt. Auch Theaterproben bestehen aus typischen Abfolgen von für die Institution spezifischen Aktivitäten, die die Arbeit am Theater konstituieren und als solche erkennbar machen (vgl. Krug et al. 2020). Aktivitäten können sich darüber hinaus zeitlich auf vergangene Aktivitäten beziehen und zukünftige Aktivitäten als mögliche Reaktionen der aktuellen Aktivität projizieren. Zum Beispiel kann innerhalb einer Besprechungsaktivität auf der Probebühne eine sich anschließende Spielaktivität relevant gesetzt werden, in der das eben Besprochene im Spiel umgesetzt wird. Daraus ergibt sich für die Teilnehmenden das praktische Problem neben der äußeren Ordnung (als Aktivitätssequenz) auch die innere Ordnung von Aktivitäten (die Handlungssequenz, mit der die Aktivität vollzogen wird) zu antizipieren. Dafür müssen Beteiligte sowohl erkennen können, wann eine Handlung im Rahmen einer Aktivität beendet sein wird und eine neue beginnt, als auch wann eine Aktivität abgeschlossen sein wird, um eine neue Aktivität initiieren zu können. Aktivitäten sind demnach zum einen in einen temporal organisierten Interaktionsfluss mehrerer aufeinanderfolgender Aktivitäten eingebettet und zum anderen durch aufeinander bezogene Beteiligungsweisen der Teilnehmenden an den sozialen Handlungen innerhalb der Aktivität strukturiert. Diese Strukturierungen sind im ethnomethodologischen Sinne methodisch.

Ein analytischer Zugriff auf soziale Aktivitäten in ihrem situativen, interaktionalen Vollzug wird mit einer konversationsanalytischen Perspektive möglich. Dafür ist es notwendig der Verwendung der Interaktionskategorie Aktivität in der konversationsanalytischen Interaktionsforschung nachzugehen und von verwandten Begriffen abzugrenzen.

\subsubsection{Aktivitäten in der Konversationsanalyse}

Obwohl der Begriff Aktivität zentral für viele konversantionsanalytische Arbeiten ist, hat eine systematische Auseinandersetzung mit dem ,activity focus“ (Drew \& Heritage 1992: 16) der Konversationsanalyse bislang kaum stattgefunden: „Even 
within Conversation Analysis, there is no clear or precise conceptualization and definition of activity, at least relative to concepts such as 'turn' and 'sequence'“ (Robinson 2013: 259). Das lässt sich damit begründen, dass der Großteil der konversationsanalytischen Studien sich mit der Organisation von Sequenzen, Turns und TCUs beschäftigt (Schegloff 2007), weshalb die Ebene der supra-sequenziellen Kohärenz (Robinson 2013: 258) bislang kaum Beachtung gefunden hat. Als Folge dieser fehlenden Auseinandersetzung lassen sich innerhalb der Konversationsanalyse zwei sich grundlegend voneinander unterscheidende Verwendungsweisen des Begriffs Aktivität beobachten: der „kleinen“ und der „großen“ Aktivitätskonzeption. Bei der „kleinen“ Aktivitätskonzeption werden Aktivitäten als Mikrophänomene verstanden: „activities of one participant, e.g., sound-stretches, hesitation phenomena, and filled pauses that project imminent self-repair" (Deppermann \& Streeck 2018: 6). Solche Aktivitäten sind grundsätzlich messbar (zählbar, zeitlich determinierbar, dokumentierbar, zeigbar, usw.). Unter dem Gesichtspunkt der Multimodalität würde damit jedes multimodale Verfahren als komplexe Sprach-Blick-Geste-usw.-Multiaktivität gefasst werden. Mit dieser Konzeptualisierung geraten demnach (Multi-)Aktivität und Multimodalität zu Synonymen, weshalb für die weitere Argumentation auf eine „große“ Aktivitätskonzeption zurückgegriffen wird. Bei dieser bildet die gemeinsame Handlungsorientierung des Interaktionsensembles (Schmitt 2012) innerhalb einer sozialen Situation (Goffman 1981) die Aktivität und ist als interaktionale Kategorie nur über eine Rekonstruktion der Beteiligungsweisen an den sozialen Handlungen der Aktivitäten nachzuweisen. Grundlegend beschreiben Pomerantz \& Fehr (2011: 169) das konversationsanalytische Verständnis von Aktivitäten, Handlungen und Praktiken als eine hierarchisch organisierte Beziehung:

Activities and actions are basic constitutive elements of interaction. People organize much of what they do in terms of activities, for example telephoning a friend, giving a lecture, or taking a break during a seminar. Generally within an activity there are one or more sequences of conversational actions. Actions refer to what the participants are doing interactionally vis- $a$-vis one another; they often are performed within turns [...]. Actions are composed of practices that provide for their intelligible enactment.

Dieser Auffassung von Aktivitäten als Rahmen für Handlungen folgt auch Goodwin (2000a: 1496) in seinen Untersuchungen, wenn er zum Beispiel das Verhältnis von Streithandlungen innerhalb einer Hüpfspielaktivität rekonstruiert:

this exchange is embedded within a larger course of action within a particular activity, playing hopscotch. [...] The structure of the encompassing activity is thus explicitly oriented to, and drawn upon as a resource for, the constitution of action within the detailed structure of the talk itself. The talk that occurs here is thus built in part through use of the resources provided by an encompassing activity, while simultaneously constituting action within it. 
Im Verlauf seiner empirischen Untersuchung zeigt Goodwin, dass a) Handlungen Teile von Aktivitäten sind, b) Beteiligte sich mit ihren Handlungen auf die übergeordnete Aktivität ausrichten und, dass c) Aktivitäten nicht nur durch Handlungen hervorgebracht werden, sondern auch zu einem gewissen Teil die notwendigen Ressourcen zur Verfügung stellen, damit Handlungen im Rahmen von Aktivitäten möglich werden. Ein solches Verständnis von Aktivitäten als Orientierungsrahmen für darin eingebettete Handlungen lässt sich beispielsweise auch in Untersuchungen zur konversationellen Aktivität GESCHICHTENERZÄHLEN (Goodwin \& Goodwin 1992) finden, bildet die konzeptuelle Basis des Handlungsverlaufs ÄRzTliche Diagnose (Heritage \& Sorjonen 1994) und liegt der Analyse der kommunikativen Aktivität VERKAUFSGESPRÄCH (Dausendschön-Gay \& Krafft 2002) zugrunde. Die genannten Studien haben neben einer ähnlichen Aktivitätskonzeption allerdings gemeinsam, dass sie Aktivitäten trotz teilweise multimodaler Erweiterung als vorrangig sprachliche Prozesse behandeln. Aus einer konsequent multimodalen Perspektive leuchtet diese Klassifizierung nicht ein, da bei multimodaler Betrachtung deutlich wird, dass selbst Aktivitäten mit hohem Sprachanteil aus mehr bestehen als allein den verbalen Beiträgen: „even prototypical speech activities such as stories, do not reside entirely, or even primarily, within the stream of speech“ (Goodwin 2018: 135). Damit entfällt die vielfach vorgenommene Unterscheidung in sprachliche und körperliche Aktivitäten und macht den Blick frei auf die multimodale Organisation bestimmter, für die jeweilige Aktivität charakteristische Elemente wie zum Beispiel syntaktische Positionen linguistischer Einheiten, prosodische Markierungen, spezielle Körperbewegungen, Blicke, Gesten, Objektmanipulation oder Referenzen auf gemeinsames Wissen. Darüber hinaus ermöglicht die multimodale Betrachtung das Erfassen einiger sonst verborgen gebliebener Beteiligungsweisen der verbalen Abstinenz (Heidtmann \& Föh 2007).

Demnach lassen sich Aktivitäten, Handlungen und Praktiken auf unterschiedlichen Granularitätsebenen (granularity, Schegloff 2000a) verorten, die sich jeweils in ihrem Grad der Auflösung (degree of resolution, Schegloff 2000a: 715) interaktionaler Phänomene und der verwendeten multimodalen Ressourcen unterscheiden. Die größte Granularitätsebene mit der niedrigsten Auflösung ist die der Aktivität (z. B. REGIEANWEISUNG). Sie bildet den Orientierungsrahmen, auf den sich die Teilnehmenden einer sozialen Situation mit ihren Handlungen ausrichten können. Innerhalb von Aktivitäten werden somit bestimmte Handlungen erwartbar. So sind die Handlungen des ${ }^{\star}$ der Regisseurs ${ }^{\star}$ in Spielinstruktion und die Instruktionsumsetzung des`der Schauspieler*in wahrscheinliche Elemente der Aktivität Regieanweisung. Auch wenn Aktivitäten damit als Handlungsrahmen konzeptualisiert werden können, handelt es sich um keine „pre-scripted routines“ (Robinson 2013: 264), sondern um situativ ausgehandelte Hervorbringungen des 
Interaktionsensembles. Als Bestandteile von Aktivitäten sind Handlungen in der Terminologie der Konversationsanalyse interaktionale Interpretationseinheiten, die sich in den Reaktionen der Beteiligten auf ein Tun eines ${ }^{\star} r$ Interagierenden offenbaren, indem sie dem Tun einen Sinn zuschreiben und auf diese Zuschreibung bezogen agieren (action formation/ascription, Levinson 2013). Es ist grundsätzlich möglich, dass einerseits ein Turn (bzw. eine TCU) mehr als eine Handlung ermöglicht, während andererseits eine Handlung über mehrere Turns (bzw. TCUs) hinweg hergestellt werden kann (Schegloff 2007: 9). Die aufeinander bezogenen Handlungen der Teilnehmenden konstituieren dabei zusammen eine laufende Aktivität (Selting 2016: 28-29). Mit dieser Perspektive sind alle Handlungen einer Aktivität eingebettete Elemente dieser Aktivität, auf die sich die Teilnehmenden im Handlungsverlauf ausrichten (Goodwin 2000c: 157). Komplexe Handlungsverläufe mit starker Vorgeformtheit und sprachlicher Verfestigung können mit (Günthner \& Knoblauch 1994) als kommunikative Gattungen bezeichnet werden. Mit Günthner (2011b: 299) beschreibt der Begriff kommunikative Gattungen „großformatige, komplexe sprachliche Gestalten (z. B. Vorlesungen, Predigten, Klatschgeschichten etc.), während der Begriff des ,Musters' für kleinere Formate (Minimal- bzw. Kleinstgattungen) verwendet wird.“ Handlungsmuster wie „eine Aufgabe festlegen“ sind in der Regel einklagbar (Jandok 2010: 189) und können von den Teilnehmenden dafür verwendet werden, „einen gemeinsamen Verständnishorizont“ (Luttermann 2011: 489) herzustellen. Handlungsmuster und kommunikative Gattungen befinden sich damit in der konzeptuellen Schnittmenge des Aktivitätskonzepts. Während die beiden erstgenannten Konzepte jedoch vor allem verfestige Handlungen und routinisierte Praktiken fokussieren, spielen bei Aktivitäten auch jene multimodalen Beteiligungsweisen eine wichtige Rolle, die keinen Handlungscharakter im engeren Sinne aufweisen, z.B. das Bilden einer f-formation (Kendon 1990) oder das Anzeigen von Verfügbarkeit in einer Interaktion (Heath 1986). Die drei Konzepte verbindet im Kern die Bedeutung von Praktiken für die Herstellung von Interaktion. Wie unter anderem Schegloff (1997: 499) herausstellt, werden Handlungen als Interpretationseinheiten der Teilnehmenden durch Praktiken realisiert. Praktiken sind dabei „routinisierte Verwendungsweisen von Ressourcen für situierte Handlungen, die flexibel an die je spezifischen Gegebenheiten angepasst wurden“ (Selting 2016: 29). Das bedeutet, dass Handlungen mit einer Reihe von Praktiken vollzogen werden können, je nachdem, welche kommunikative Aufgabe für die Interagierenden ansteht. Um beispielsweise im Rahmen der Aktivität REgIEANWEISUNG die Handlung Spielinstruktion zu realisieren, können Regieführende auf Praktiken des Vor- oder Mitspielens zurückgreifen. Das bedeutet, dass je nachdem, welche Praktiken realisiert werden, andere Handlungen (und damit unter Umständen auch andere Aktivitäten) für die Beteiligten erkennbar werden: „practices, deployed always in some position, can accomplish 
different actions; and actions can be accomplished through a variety of situated practices“ (Schegloff 1997:505). Auf der untersten Granularitätsebene mit der höchsten Auflösung in Bezug auf interaktionale Phänomene sind die (multimodalen) Ressourcen zu verorten, mit denen Praktiken vollzogen werden, durch die bestimmte Handlungen innerhalb situierter Aktivitäten erkennbar werden. So sind es bestimmte prosodische (z. B. erhöhe Stimmlage), verbale (z. B. Realisation von Stücktext) und gestische (z. B. erhobene Hände) Ressourcen, mit denen eine Vorspiel-Praktik als Initiierung der Handlung Spielinstruktion im Rahmen einer Aktivität REGIEANWEISUNG vollzogen werden kann (vgl. Wessel 2020).

Die granulare Konzeption von Aktivitäten als aufeinander bezogene Sammlung von Handlungen und Praktiken, mit denen diese hergestellt werden, deckt einen Großteil vieler Partizipationsmöglichkeiten an Aktivitäten ab. Jedoch muss nicht jede Beteiligungsweise eine Handlung sein. Deutlich wird dies vor allem mit einer multimodalen Perspektive auf Interaktion, wenn beispielsweise verbal abstinente Teilnehmende zwar dem Verlauf einer Aktivität folgen, aber nicht zwangsläufig eigene Handlungen realisieren müssen (Heidtmann \& Föh 2007). Hinweise darauf, dass verbal abstinente Beteiligungsweisen durchaus interaktionale Relevanz für Interagierende haben, zeigen etwa die Analysen zur Äußerungsemergenz bei un/wissenden Adressaten (Goodwin 1979), zu Nicken als Involvierungsmarker bei fehlendem Rezipienten-Blickkontakt (Goodwin 1984) oder zur Antizipation des Sprecherwechsels durch nicht-adressierte Dritte (Holler \& Kendrick 2015). Nicht-Sprechende sind damit nicht nur Zuhörende, sondern stellen mit ihren multimodalen Beteiligungsweisen den Rahmen für die laufende Aktivität her (Goodwin 2018: 50). Darüber hinaus können verbal abstinente Teilnehmende in „silent activities“ (Deppermann \& Streeck 2018: 16; Mondada 2019b) involviert sein, die mit der laufenden sprachgebrauchgebundenen Aktivität koordiniert werden muss. Monitoring der laufenden Interaktion und das damit einhergehende Anzeigen von Zuhörerschaft (Heath 1986) als doing being an activity's member (Sacks 1992) dient somit als zentrale Beteiligungsweise, mit der Beteiligte ihren Teilnehmendenstatus (Goffman 1963, 1979; Goodwin 2000b, 2007; Goodwin \& Goodwin 2004) in Bezug auf die aktuelle(n) Aktivität(en) anzeigen können. Beteiligung ist in diesem Sinne keine Typologie von Kategorien wie die Goffman'schen (1981) Interagierendenpositionen des Beteiligungsrahmens (wie ratified participants, bystander, eavsdropper, etc), sondern ein sich mit dem Verlauf der jeweiligen Aktivität stetig verändernder Prozess der wahrnehmbaren Orientierung und Teilhabe an der jeweiligen Aktivitäten (Goodwin 2018: 135).

Für den weiteren Verlauf dieser Arbeit stehen daher nicht die Aktivitäten konstituierenden sozialen Handlungen oder deren Handlungsmuster im Fokus, sondern die multimodal organisierten Beteiligungsweisen, mit denen Teilnehmende ihre Involvierung in mehr als eine Aktivität zur gleichen Zeit gegenseitig 
anzeigen. Dies ermöglicht es auch jene sprachfreien Verfahren zu beschreiben, auf die Teilnehmende bei der Koordination mehrerer gleichzeitig relevanter Aktivitäten zurückgreifen, die analytisch als Handlungen schwerer fassbar sind (vgl. Kap. 3). Indem das Phänomen der gleichzeitigen Relevanz multipler Aktivitäten nicht aus der Handlungs-, sondern der Beteiligungsperspektive betrachtet wird, werden nicht nur die handelnden Teilnehmenden, sondern alle Mitglieder des Interaktionsensembles, die ihre Beteiligungsweisen auf die ko-relevanten Aktivitäten ausrichten, als Koordinierende innerhalb einer sozialen Situation erkennbar. Das erlaubt einen analytischen Zugriff auf die strukturelle (In)Kompatibilität der Beteiligungsweisen an den ko-relevanten Aktivitäten durch die Teilnehmenden. Die gleichzeitige Relevanz multipler Aktivitäten umfasst damit nicht zwangsläufig das zeitgleiche Vollziehen mehrerer Handlungen, sondern drückt sich darin aus, dass Teilnehmende ihre Beteiligungsweisen so organisieren, dass sie Involvierung in zwei (oder mehr) ko-relevante Aktivitäten anzeigen können.

\subsubsection{Aktivitätskategorien der intersubjektiven Gestaltung: Ensemble- und Einzelaktivitäten}

Aktivitäten sind soziale Einheiten, die sich in ihrer Organisation maßgeblich in ihren Beteiligungsweisen unterscheiden, mit denen sie hergestellt werden. In der Situation, die bereits in der Einleitung in Transkript A betrachtet wird, zeigt sich dies bei der gemeinsamen Aushandlung der Theaterführung durch die Regieassistentin und die Hospitantin. Abbildung A.3 zeigt einen Moment dieser Interaktion, in dem die Regieassistentin ISST, die Hospitantin ihren MANTEL ABLEGT und beide miteinander im Zuge des gemeinsamen WISSENSABGLEICHS sprechen, während sie ihre Körper aufeinander ausgerichtet haben und sich vermutlich gegenseitig anschauen.

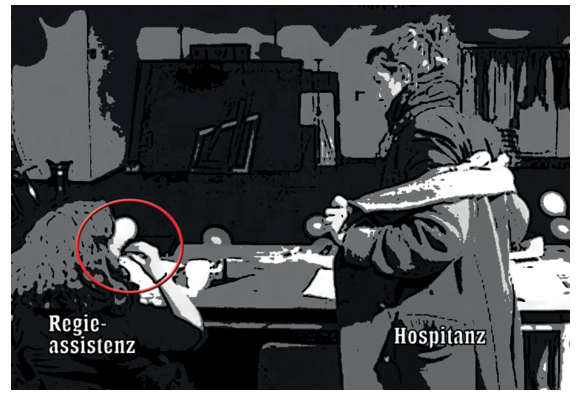

Abb. A.3: ASS ISST eine Nuss (Kreis). HOS LEGT DEN MANTEL AB.

Bei allen drei Aktivitäten (WiSSENSABgleich, ESSEN, MANTEL ABLEGEN) handelt es sich um soziale Einheiten, weil sie in der Reicheichweite einer anderen Akteu- 
rin (,in one another's response presence“, Goffman 1983: 2) hervorgebracht und auf diese ausgerichtet werden - auf Abb. A.3 gut zu erkennen anhand der gegenseitigen Körper- und Kopforientierung. Die Aktivitäten EsSEN und MANTEL ABLEGEN unterscheiden sich vom WISSENSABGLEICH darin, wie stark deren Beteiligungsweisen auf die Ko-Interagierende orientiert ist. Um zu ESSEN, benötigt die Regieassistentin in der Situation keine Beteiligung einer anderen Person an dieser Aktivität. Auch die Hospitantin ist die einzige Interagierende, deren haptische Beteiligungsweise in diesem Moment die Aktivität MANTEL ABLEGEN herstellt. Im Gegensatz dazu kommt die gemeinsame Aktivität WISSENSBESTÄNDE ABGLEICHEN dadurch zustande, dass die Interagierenden sich mittels verbaler (z. B. Frage-Antwort-Paarsequenzen) und visuell-proxemischer (z. B. Körperorientierung aufeinander, Blickkontakt) Beteiligungsweisen gegenseitig ihre Involvierung in die gemeinsame Aktivität anzeigen und sie intersubjektiv mit ihren aufeinander bezogenen Interaktionsbeiträgen vollziehen. Im Folgenden werden solche intersubjektiv ausgehandelten Aktivitäten eines Interaktionsensembles als Ensembleaktivität bezeichnet. Ensembleaktivitäten genießen in der interaktionsanalytischen Forschungslandschaft eine zentrale Stellung. Unabhängig von der Ausrichtung auf verbale Strukturen des kollektiven Erzählens (Quasthoff 1980) bzw. des gemeinsamen Sprechens (Schwitalla 1993) oder auf eher multimodale Perspektiven der Ko-Konstruktionen von Handlungen (Dausendschön-Gay, Gülich \& Krafft 2015) liegt der Forschungsschwerpunkt stets auf der gemeinsamen interaktiven Herstellungsleistung mehrerer Interagierender (Schegloff \& Sacks 1973). Mit der Konzeption von Ensembleaktivität als von einer (mindestens dyadischen) Teilnehmendenkonstellation hergestellten, intersubjektiv organisierten Aktivität soll genau jener Aspekt betont werden. Die Teilnehmendenkonstellation Ensembleaktivität ist also dadurch kennzeichnet, dass mehrere Beteiligungsweisen von mindestens zwei verschiedenen Interagierenden eines Interaktionsensembles vollzogen werden. Beteiligungsweisen meint im Rahmen dieser Studie die Verfahren, mit denen Beteiligte auf eine Interaktion einwirken und Involvierung anzeigen können. Dies umfasst potentiell alle aufeinander bezogenen, interpersonell koordinierten Prozesse, die im Rahmen der multimodalen Konversationsanalyse typischerweise betrachtet werden wie verbale, gestische, mimische, visuelle, proxemische oder haptische Beteiligungsweisen, um nur einige zu nennen.

Die Aktivitäten MANTEL ABLEGEN oder ESSEN werden in der oben beschriebenen Situation jeweils nur durch die Beteiligungsweisen einer einzigen Teilnehmenden aufrechterhalten und erfordern keine intersubjektive Aushandlung. Solche Aktivitäten werden folgend als Einzelaktivität kategorisiert. Einzelaktivitäten haben aufgrund der Fokussierung auf verbale Phänomene im Gegensatz zu Ensembleaktivitäten bislang nur selten Einzug in konversationsanalytische Arbeiten gefunden. Wenn sie bisher betrachtet worden sind, wurde ihr Auftreten 
oft als „silent activities“ (Deppermann \& Streeck 2018: 16) beschrieben. Dies kann eine sinnvolle Kategorisierung sein, wenn der Fokus auf verbalen Phänomenen liegt. Wenn hingegen aus multimodaler Perspektive die soziale Konstitution von (multiplen) Aktivitäten untersucht werden soll, ist diese verbal-zentrierte Einteilung nicht zielführend - zumal auch Objektübergaben als intersubjektiv gestaltete Aktivitäten zwischen zwei Interagierenden problemlos sprachfrei verlaufen können (Due \& Trærup 2018; Mondada 2019b). Die häufigste Konzeptualisierung der individuellen Aktivitätsform als soziale Einheit erfolgt in der Forschungsliteratur als „private Aktivität“ (Goodwin 1981; Mortensen 2013; Mondada 2008). Dort werden sie analytisch nicht selten als „non-interactional activities [...] such as 'working for oneself'“ (Koole 2007: 489) behandelt. Da Aktivitäten in der für diese Arbeit geltenden Konzeption immer sozial organisiert sind, wird statt „stiller“, „privater“ oder „individueller“ Aktivität der Begriff Einzelaktivität verwendet. Einzelaktivitäten werden nur durch Beteiligungsweisen einzelner Beteiligter in ständiger Koordinierung mit anderen laufenden Aktivitäten der sozialen Situation realisiert. Eine intersubjektive Organisation der Einzelaktivität ist dabei aber im Gegensatz zu Ensembleaktivitäten nicht erkennbar. So wie sich bei der Organisation von Ensembleaktivitäten Handlungsphasen (z. B. erster Paarteil und zweiter Paarteil einer Paarsequenz) finden lassen, so wird mithilfe des Gestalt-Konzepts die phänomenologische Form verschiedener Phasen einer Aktivität als Koordinationsprodukt mehrerer multimodaler Ressourcen beschreibbar. Goodwin (1981: 109) zeigt dies anhand der Einzelaktivität BIER-TRINKEN innerhalb einer Interaktion. Dort wird die Aktivität durch das Heranführen des Gefäßes an den Mund zwar gestartet (Aktivitätsstart), aber durch eine Frage eines anderen Interaktanten abgebrochen, sodass sofort zum Aktivitätsende übergegangen wird, ohne den Aktivitätskern (die eigentliche Handlung Trinken) auszuführen. In Goodwins Beispiel wird deutlich, dass die Aktivitätsphasen für die Interagierenden interaktional relevant sind: Da während des Trinkvorgangs selbst nicht gesprochen werden kann, wird die Aktivität des TRINKENs zugunsten einer anderen Aktivität beendet. Auch bei Hoey (2018a) lassen sich diese Aktivitätsphasen als „preparation phase“ (=Startphase), „focal action phase“ (=Kernphase) und „return phase“ (=Endphase) wiederfinden. Die Position, aus der die Aktivitäten starten und nach Abschluss wieder zurückkehren, kann als „home position“ (Schegloff 1998; Sacks \& Schegloff 2002) gefasst werden. Diese Bezeichnungen, die an die Kendon'schen Gestenphasen (Kendon 1980) angelehnt sind, beschreiben primär proxemische Trajektorien, wie sie bei vielen Einzelaktivitäten beobachtbar sind. Aktivitäten ohne solche proxemische Ressourcen können damit aber nicht erfasst werden, weshalb im Folgenden die allgemeineren Termini Start-/Kern-/Endphase Verwendung finden, da sie sowohl für Einzel- wie auch Ensembleaktivitäten angewendet werden können. 
Die Kategorisierung von Aktivitäten in Bezug auf ihre intersubjektive Organisation ist nicht fest determiniert, sondern kann im interaktionalen Verlauf verändert werden. Dementsprechend können Aktivitäten, die in einer Situation als Einzelaktivitäten realisiert werden, in einer anderen Situation durchaus auch als Ensembleaktivitäten vollzogen werden. So ist beispielsweise denkbar, dass ESSEN als Ensembleaktivität FÜTTERN von zwei Interagierenden realisiert wird (z. B. in Eltern-Kind-Interaktionen) und MANTEL ABLEGEN als Ensembleaktivität in Unterstützungssituationen relevant werden kann. Einen Beleg dafür, dass Interagierende ihre Aktivitäten je nach situativer Anforderung in der intersubjektiven Gestaltung anpassen, zeigt Mortensen (2013) anhand des VORLESENDEN SCHREIBENS (writing aloud) bei einer Gruppendiskussion in einem Meeting. Dort modifizierenden Interagierende die sonst typischerweise als Einzelaktivität realisierte Aktivität ScHREIBEN als intersubjektiv ausgehandelte Ensembleaktivität, indem sie haptische mit verbalen Beteiligungsweisen kombinieren. Dies ermöglicht es den anderen Interagierenden, den Fortschritt des ScHREIBENS zu verfolgen und ihre eigenen Beteiligungsweisen darauf auszurichten. Analog dazu beschreiben Heuser, Arend \& Sunnen (2020) VoRLESEN (reading aloud) als eine Aktivität, bei der ein sonst häufig als Einzelaktivität auftretender Vorgang (LESEN) durch Vokalisation zur Ensembleaktivität ausgebaut wird und dadurch Partizipationsmöglichkeiten für andere Interagierende eröffnet. Ein ähnlich gelagertes Phänomen findet sich bei Mondada (2011), wenn Chirurgen Einzelaktivitäten wie OPERIEREN dadurch für andere Beteiligte als Ensembleaktivität intersubjektiv verfügbar machen, dass sie ihre Assistenten*innen zu bestimmten operationsrelevanten Handlungen auffordern und daraufhin der Eingriff kollaborativ vollzogen wird. Damit wird deutlich, dass Aktivitäten in Abhängigkeit von der situativen Anforderung als Ensemble- oder als Einzelaktivitäten organisiert werden können. Dies hat nicht nur Einfluss darauf, ob bzw. wie andere Beteiligte ihre Beteiligungsweisen auf diese Aktivitäten ausrichten, sondern auch auf die koordinativen Praktiken, mit denen Interagierende Einzel- und Ensembleaktivitäten simultan mit anderen Aktivitäten abstimmen.

Die vorgeschlagene Kategorisierung von Aktivitäten in Bezug auf ihre intersubjektive Aushandlung ist insofern für die Betrachtung der Koordinationspraktiken multipler Aktivitäten sinnvoll, als Beteiligte multiple Aktivitäten in Abhängigkeit von den Beteiligungsweisen anderer Interagierender koordinieren. Wie sich in den Analysekapiteln 5 bis 7 zeigen wird, unterscheidet sich die Koordination mehrerer Einzelaktivitäten von einer Koordination verschiedener Ensembleaktivitäten vor allem in der intersubjektiven Gestaltung der Beteiligungsweisen, was sich in der strukturellen Kompatibilität der verwendeten Beteiligungsweisen ausdrückt. 